\title{
Continuous wavelet transform and neural network for condition monitoring of roto- dynamic machinery
}

\author{
T Kaewkongka, Y H Joe Au, R Rakowski and B E Jones \\ The Brunel Centre for Manufacturing Metrology, \\ Brunel University, Uxbridge, \\ Middlesex UB8 3PH, UK \\ Phone: (+44) 01895274000 ext. 2608, E-mail: joe.au@brunel.ac.uk
}

\begin{abstract}
This paper describes a novel method of rotodynamic machine condition monitoring using a wavelet transform and a neural network. A continuous wavelet transform is applied to the signals collected from accelerometer. The transformed images are then extracted as unique characteristic features relating to the various types of machine conditions. In the experiment, four types of machine operating conditions have been investigated: $a$ balanced shaft, an unbalanced shaft, a misaligned shaft and a defective bearing. The back propagation neural network (BPNN) is used as a tool to evaluate the performance of the proposed method. The experimental results result in a recognition rate of 90 percent.
\end{abstract}

Keywords - Wavelet transform, neural network, rotodynamic machinery.

\section{INTRODUCTION}

With ever growing competition in industry, the need for machine condition monitoring has become more important. A reliable condition monitoring system will significantly reduce failure and unplanned maintenance, and hence the huge attendant cost due to machine downtime. Often, the system is used with an operator who assists in the interpretation of the machine signals for early failure detection and fault diagnosis.

Nowadays there are two kinds of methods available for bearing maintenance: statistical bearing life estimation and bearing condition monitoring and diagnostics [1]. Statistical bearing life estimation predicts the fatigue life of a bearing. However, its application has many limitations, since unusual operating conditions can severely decrease a bearing's life. Bearing life estimates become unreliable leading to unexpected breakdown. On the other hand, bearing condition and diagnostics can be a very reliable method because it gives upto-date information about the condition of a bearing. The more popular techniques used for bearing condition monitoring are vibration and acoustic emission analyses.

\section{PREVIOUS WORK}

Currently, there are many conventional methods for identifying and diagnosing bearing faults. Based on the representation of a signal during its processing, a method can be referred to as time-domain or frequency-domain.
Time domain methods are usually sensitive to impulsive oscillations. Characteristics arising from the defects being monitored, also known as features, of the raw time signal can be extracted from a machine. Typical features are the r.m.s. value, peak value [2], crest factor, kurtosis [3] and the shape, size and orientation of a bearing locus derived from orbital analysis. These features, once established to be related to the defect being monitored, often yield satisfactory results. However, if the signal generation mechanism is complex, time-domain methods are often not refined enough.

Frequency domain methods assume that the signal being analyzed has components that are periodic. Thus, a defect produces a periodic signal at the characteristic defect frequency. Examples of the frequency-domain methods include spectrum analysis, cepstrum analysis, high frequency resonance technique (HFRT) [4] and holospectrum [5]. Among them, spectrum analysis seems to dominate the fault diagnosis scene. The main limitation of spectrum analysis is that although a local transient will contribute towards the overall frequency spectrum, its location on the time axis is lost. There is no way of knowing whether a particular frequency component exists throughout the life of the time signal or during just one or a few selected periods. Unfortunately, many monitoring situations demand knowledge of not just the frequency composition of a transient but also its time of occurrence. For instance, when a rolling element passes a localized defect in a bearing, it generates a transient in the measured signal, as does the contact of a damaged tooth with other teeth in a gearbox. A machine with rapidly varying speed is another example of transient events.

The continuous wavelet transform (CWT), a joint time- and frequency-domain technique, is proposed in this paper. CWT is capable of indicating abrupt changes in machine conditions [6]. In addition, it can give a better representation of the signal than conventional methods, providing fuller information on the machine operating condition. CWT is used here to produce a 3-D image from the measured signal. Features are then extracted from the image to be used as inputs to a back propagation neural network, the tool used for assessing the discriminatory power of CWT. 


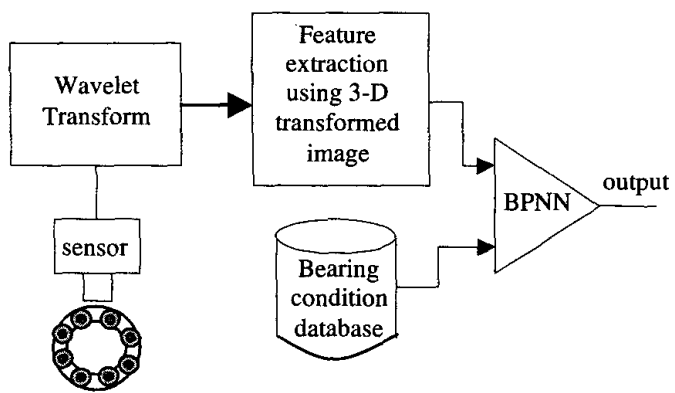

Fig. 1. The proposed condition monitoring of rotodynamic machinery block diagram.

\section{METHODOLOGY}

The proposed method of machine bearing faults recognit (Fig. 1), using continuous wavelet transform and ba propagation neural network consists of three steps.

Step 1 Applying continuous wavelet transform

Continuous wavelet transform of a time-signal $f(t)$ is defined by [7]

$$
F_{\Psi}(a, b)=\frac{1}{\sqrt{a}} \int_{-\infty}^{\infty} f(t) \Psi\left(\frac{t-b}{a}\right) d t
$$

The quantity $\Psi_{a, b}(t)=\frac{1}{\sqrt{a}} \Psi\left(\frac{t-b}{a}\right)$ given in the definition is referred to as the wavelet function. The position variable $b$ shifts the wavelet function along the time axis $t$ of $f(t)$ while the scale variable $a$ expands or compresses the wavelet function $\Psi_{a, b}(t)$. Compared to Fourier transform, the scale variable $a$ is equivalent to the inverse of the frequency. The definition also suggests that $F_{\psi}(a, b)$ is the correlation coefficient between the wavelet function $\Psi_{a, b}(t)$ and the time-signal $f(t)$ at the scale $a$ and position $b$ of $\Psi_{a, b}(t)$.

The continuous wavelet transform was applied to the acceleration signals from the four machine conditions: (a) balanced shaft, (b) unbalanced shaft, (c) misaligned shaft and (d) defective bearing. Samples of these signal are as shown in Fig. 2. CWT was applied to these signals to calculate the coefficients $F_{\Psi}(a, b)$, which were then displayed as a grey-scale map with the vertical and horizontal axes denoting respectively the scale $a$ and position $b$, which is equivalent to time $t$, as shown in Fig. 3 for the four different conditions. Although there is general similarity between them, subtle differences are clearly visible.
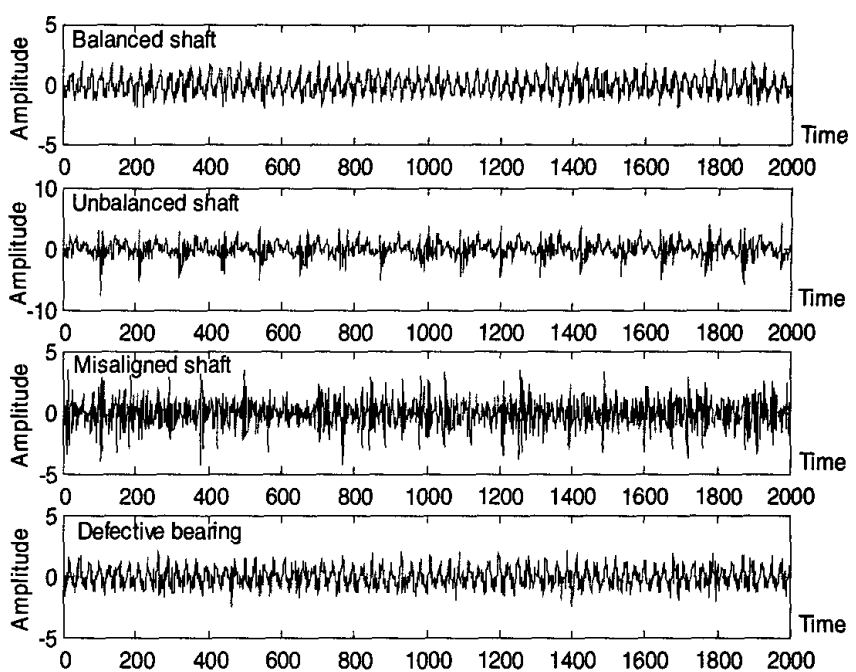

Fig. 2. Acceleration signals from the four bearing conditions

Step 2 Preprocessing

Image preprocessing was performed using the method of thresholding or binarising [8]. It was applied to the greyscale CWT image to convert the colour of each pixel into either black or white.

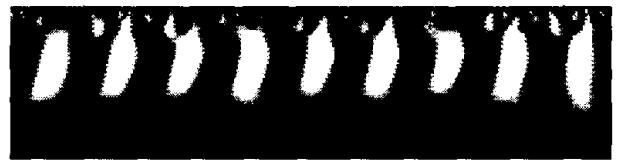

(a)

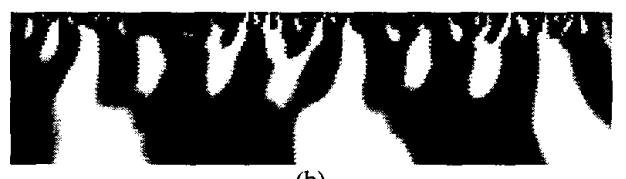

(b)

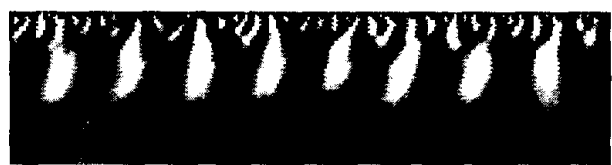

(c)

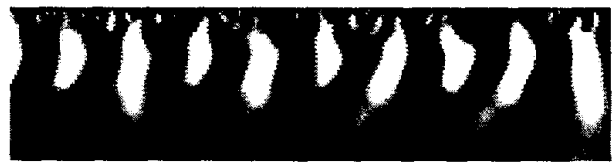

(d)

Fig. 3. Transformed images of (a) Balanced shaft, (b) Unbalanced shaft, (c) Misaligned shaft and (d) Defective bearing 

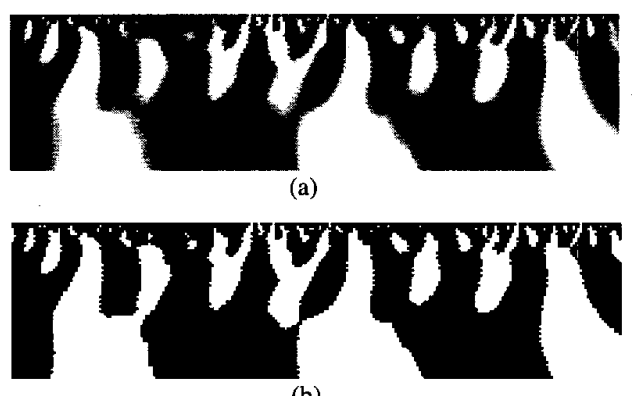

(b)

Fig. 4. The original image (a) and its binary image (b)

A binary image has the obvious advantage that when classification using neural networks is done, the computation time will be much shorter, as multiplication involving a 0 or 1 is much easier to perform. Fig. $4 \mathrm{a}$ and $4 \mathrm{~b}$ show a sample image before and after binarising.

\section{Step 3 Classifying}

The back-propagation neural network (BPNN) [9], with the architecture as shown in Fig. 5, was used to classify bearing faults. The network has an input layer, a hidden layer and an output layer. The values of the pixels composing the CWT binary image provided the inputs to the neural network; and 4 nodes, representing the bearing conditions of normal, unbalanced, misaligned and defective bearing were available as outputs.

Through a process of trial and error based on minimizing the mean square error (MSE), the choice of 12 nodes in the first hidden layer and 18 nodes in the second hidden layer were determined to be optimal.

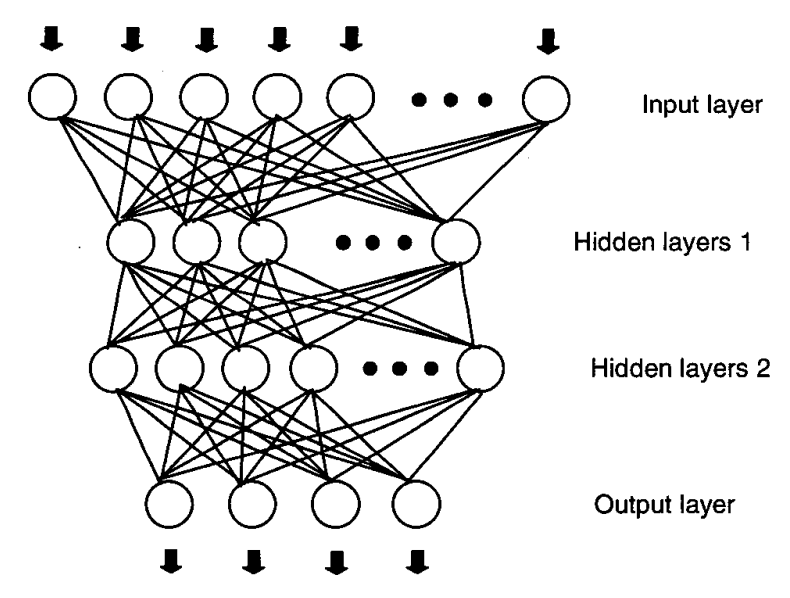

Fig. 5. BPNN architecture

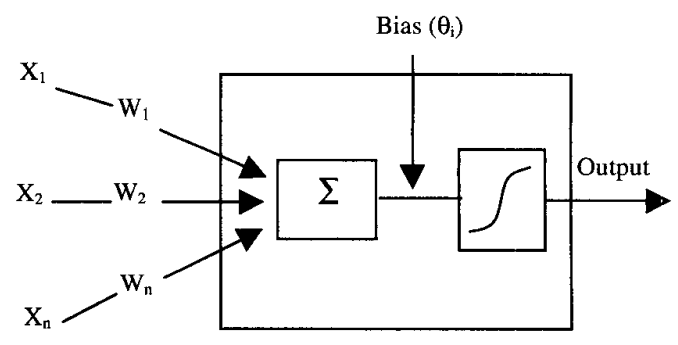

Fig. 6. Schematic process of BPNN

The value NET appearing at a node in a layer is computed by summing the products of all inputs leading to that node with their corresponding weights plus a bias. This value then forms the argument of a transfer function $f$ that produces an output for the node. In vector notation, given the input vector $X_{i}$, the weight vector $W_{i}$, and the bias vector $\theta_{i}$, the output is given by

$O U T=f(N E T)=f\left(\sum_{i}^{n} X_{i} W_{i}+\theta_{i}\right)$

The transfer function $f$ is the commonly used sigmoid function defined as

$$
f=\frac{1}{1+e^{(-N E T)}}
$$

The sigmoid function acts as an output gate that can be opened $(0)$ or closed (1). The computing process as described is schematically shown in Fig. 6.

The Back-propagation algorithm is used to obtain the correct weights and biases in a training process. A set of training data with known outputs is fed into the network. The weights are initially set to random values; the biases are fixed at unity. The input data are presented to the network; outputs are calculated and compared with the desired outputs. The normalized mean square error (MSE) is then calculated and propagated back to adjust the weights on the neural connection. This process is repeated for a large number of epochs until the error is relatively low and acceptable, which allows the network to classify the test set correctly.

\section{EXPERIMENTAL VERIFICATION OF THE PROPOSED METHOD}

Experiments were conducted on a rotodynamic test rig, Fig. 7 , consisting of a rotating shaft driven by a DC motor at 20 rev/sec. The shaft, supported in two bearings, carried 4 discs with attachable masses in order to produce rotating unbalance. The two bearings were a FAG 20205K.T.C3 self- 


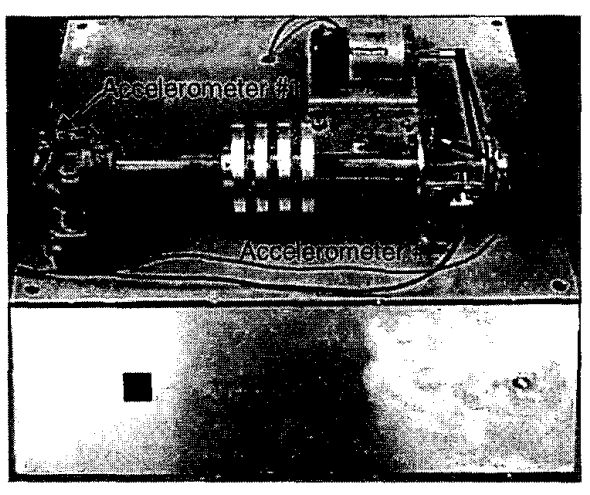

Fig. 7. Test rig set-up

aligning single row taper-bore bearing and an FAG 6304 ball bearing. Radial vibration at the two bearings was measured using two PCB 333A12 accelerometers, one on top of the non-drive end housing while the other on top of the drive-end housing labeled as \#1 and \#2 respectively in Fig. 7. The acceleration signals, having been low-pass filtered at $1 \mathrm{kHz}$ for anti-aliasing, were sampled into a Labview data acquisition system.

Measurements were obtained from four different machine conditions: balanced shaft, unbalanced shaft, misaligned shaft and defective bearing. For each condition, twenty signals were collected which were divided into two equal sets of ten each. The first set was used for training the neural network while the second set was used for testing it.

As mentioned in Step 2, it was the binarised CWT image of a signal that was being used in training and testing. The image consisted of 64 scales and 250 time intervals giving a resolution of $64 \times 250=16000$ pixels.

\section{RESULTS AND DISCUSSION}

Classification results of 40 events from the accelerometer signals of all four types of machine conditions are given in Table I. It shows the output value from the back propagation neural network after the network has been trained. For the similar pattern, the output value is around unity, rather than zero. In contrast, for a poor match, the value returned is around zero.

To examine the results, the maximum output is used to identify the bearing condition. The symbols in the second column from the left indicate the following:

$\mathrm{R}=$ classified correctly

$\mathrm{W}=$ misclassified
Table I

Correlation coefficient results

\begin{tabular}{|c|c|c|c|c|c|}
\hline $\begin{array}{c}\text { Bearing } \\
\text { conditions }\end{array}$ & \multicolumn{5}{|c|}{ Training sets } \\
\hline Test sets & & $\begin{array}{c}\text { Balance } \\
\text { shaft }\end{array}$ & $\begin{array}{c}\text { Unbalanced } \\
\text { shaft }\end{array}$ & $\begin{array}{c}\text { Misaligned } \\
\text { shaft }\end{array}$ & $\begin{array}{c}\text { Defective } \\
\text { bearing }\end{array}$ \\
\hline \multirow{10}{*}{ Balance shaft } & W & 0.22 & -0.01 & 0.00 & 0.79 \\
\hline & $\mathbf{R}$ & 0.94 & 0.00 & 0.00 & 0.06 \\
\hline & $\mathbf{R}$ & 0.36 & 0.19 & 0.00 & 0.34 \\
\hline & $\mathrm{R}$ & 0.92 & -0.08 & 0.00 & 0.23 \\
\hline & $\mathrm{R}$ & 0.98 & 0.00 & 0.00 & 0.03 \\
\hline & $\mathbf{R}$ & 0.96 & 0.03 & 0.03 & -0.03 \\
\hline & $\mathrm{R}$ & 0.86 & -0.02 & 0.00 & 0.18 \\
\hline & $\mathrm{R}$ & 0.73 & 0.00 & 0.00 & 0.27 \\
\hline & W & 0.20 & -0.01 & 0.00 & 0.81 \\
\hline & $\mathrm{R}$ & 0.98 & 0.05 & 0.01 & -0.02 \\
\hline \multirow{10}{*}{$\begin{array}{c}\text { Unbalanced } \\
\text { shaft }\end{array}$} & $\mathrm{R}$ & 0.04 & 0.35 & 0.22 & 0.26 \\
\hline & $\mathrm{R}$ & 0.04 & 1.00 & 0.00 & -0.04 \\
\hline & $\mathrm{R}$ & -0.12 & 0.84 & 0.00 & 0.45 \\
\hline & $\mathbf{R}$. & 0.01 & 0.89 & 0.00 & 0.08 \\
\hline & $\mathrm{R}$ & -0.01 & 0.97 & 0.00 & 0.04 \\
\hline & W & -0.01 & 0.19 & 0.29 & 0.47 \\
\hline & $\mathrm{R}$ & 0.00 & 1.01 & 0.00 & -0.01 \\
\hline & $\mathrm{R}$ & -0.08 & 0.92 & 0.01 & 0.18 \\
\hline & $\mathbf{R}$ & 0.1 & 0.70 & 0.04 & 0.09 \\
\hline & $\mathrm{R}$ & -0.01 & 1.05 & 0.00 & -0.05 \\
\hline \multirow{10}{*}{$\begin{array}{l}\text { Misaligned } \\
\text { shaft }\end{array}$} & $\mathbf{R}$ & 0.00 & 0.00 & 0.99 & 0.00 \\
\hline & $\mathrm{R}$ & 0.00 & 0.00 & 1.00 & 0.00 \\
\hline & $\mathrm{R}$ & 0.01 & 0.07 & 0.95 & 0.12 \\
\hline & $\mathbf{R}$ & 0.00 & 0.02 & 1.00 & -0.02 \\
\hline & $\mathrm{R}$ & 0.00 & 0.00 & 1.00 & 0.01 \\
\hline & $\mathbf{R}$ & 0.01 & -0.03 & 0.95 & 0.05 \\
\hline & $\mathrm{R}$ & 0.00 & 0.00 & 1.00 & 0.00 \\
\hline & $\mathbf{R}$ & 0.00 & 0.00 & 0.99 & 0.00 \\
\hline & $\mathbf{R}$ & 0.00 & 0.00 & 0.99 & 0.00 \\
\hline & $\mathbf{R}$ & 0.00 & 0.04 & 1.00 & -0.04 \\
\hline \multirow{10}{*}{$\begin{array}{c}\text { Defective } \\
\text { bearing }\end{array}$} & $\mathbf{R}$ & -0.01 & 0.08 & 0.33 & 0.51 \\
\hline & $\mathrm{R}$ & 0.02 & 0.01 & 0.00 & 0.94 \\
\hline & $\mathbf{R}$ & -0.08 & -0.10 & 0.10 & 1.10 \\
\hline & $\mathrm{W}$ & 0.30 & 0.34 & -0.02 & 0.30 \\
\hline & $\mathbf{R}$ & -0.02 & 0.13 & 0.26 & 0.60 \\
\hline & $\mathbf{R}$ & -0.05 & 0.00 & 0.42 & 0.67 \\
\hline & $\mathbf{R}$ & 0.24 & -0.02 & 0.00 & 0.78 \\
\hline & $\mathrm{R}$ & -0.09 & 0.32 & 0.29 & 0.52 \\
\hline & $\mathrm{R}$ & 0.00 & -0.05 & 0.44 & 0.64 \\
\hline & $\mathbf{R}$ & 0.38 & -0.10 & -0.02 & 0.88 \\
\hline
\end{tabular}


The signals were classified correctly 36 times out of 40 with a recognition rate of 90 percent. However, due to the fact that an intensity pixel being less than the threshold value had to be omitted, this brought down the recognition rate. Therefore, if an extracted image consists of many light intensity pixels, it may not find enough significant intensity pixels to represent the characteristic features efficiently. In other words, the shape of the extracted image is distorted by the lack of pixels which contain the unique pattern for each bearing condition. Therefore, this may lead to incorrect results.

\section{CONCLUSIONS}

Condition monitoring of rotodynamic machinery has been investigated by using the continuous wavelet transformed image as a characteristic feature of each signal condition and the back propagation neural network as a classification tool. Results are summarised below:

1) A major contribution of this work is the introduction of a new feature extraction method and feature representation for condition monitoring using continuous wavelet transform. Image processing techniques have been employed to remove the broadband noise from the signal before the final stage of classification is performed using a back-propagation neural network.

2) The wavelet transform has the main advantage that it provides information of the signal on scale (frequency) as well as on time. The transformed image therefore contains information in both time and frequency which enhances the ability to discriminate properly between the four types of bearing condition.

3) The classification error is due to the omission of some pixels by binarising and it is dependent on the threshold value. It is believed that if the setting of the threshold level is optimised, a possible higher recognition rate would be achieved.

\section{ACKNOWLEDGMENTS}

The authors wish to gratefully acknowledge the Royal Thai government for its support, also thank Corus, Middlesborough UK, which kindly provided material help and advice for experimentation, the support of EPSRC (Grant GR/M44200) and the nine industrial collaborators including the UK National Physical Laboratory within the INTErSECT Faraday Partnership Flagship Project (1998-2002) entitled "Acoustic Emission Traceable Sensing and Signature Diagnostics(AESAD)"'(Proect website:

http://www.brunel.ac.uk/research/bcmm/aesad/).

\section{REFERENCES}

[1] Y. Li, S. Billington, C. Zhang, T. Kurfess, S. Danyluk and S. Liang, "Adaptive prognostics for rolling element bearing condition," Mechanical Systems and Signal Processing, Vol.13(1), pp.103-113, 1999.

[2] S. Zhang, R. Ganesan, and G. D. Xistris, "Self-organizing neural net works for automated machinery monitoring systems," Mechanical Systems and Signal Processing, Vol.10(5), pp. 517-532,1996.

[3] C. Pachaud, R. Salvetat and C. Fray, "Crest factor and kurtosis contribu tions to identify defects inducing periodical impulsive forces," Mechanical Systems and Signal Processing, Vol.11(6), pp. 903-916, 1997.

[4] J. Shiroishi, Y. Li, S. Liang, T. Kurfess and S. Danyluk, "Bearing con dition diagnostics via vibration and acoustic emission measurement," Mechanical Systems and Signal Processing,Vol.11(5), pp. 693-705, 1997.

[5] L. Qu, X. Liu, G. Peyronne and Y. Chen, "The Holospectrum: A new method for rotor surveillance and diagnosis," Mechanical Systems and Signal Processing, Vol.3(3), pp. 255-267, 1989.

[6] H. Suzuki, T. Kinjo, Y. Hayshi, M. Takemoto and K. Ono, "Wavelet transform of acoustic emission signals," Journal of Acoustic Emission, Vol.14(2), pp. 69-84, 1996.

[7] L. Gaul and S. Hurlebaus, "Identification of the impact location on a plate using wavelets," Mechanical Systems and Signal Processing, Vol.12(6), pp. 783-795, 1997.

[8] Rafael C. Gonzales, Richard E. Woods, Digital Image Processing, (Addison Wesley), 1993.

[9] B. A. Paya and I. I. Esat, "Artificial neural network based fault diagnos tics of rotating machinery using wavelet transforms as a preprocessor," Mechanical Systems and Signal Processing, Vol.11(5), pp. 751-765, 1997. 